\title{
Products of an arbitrary number of Hecke eigenforms
}

by

\author{
Brad A. Emmons (Utica, NY) and \\ Dominic Lanphier (Bowling Green, KY)
}

1. Introduction. Let

$$
E_{k}(z)=1+\frac{(2 \pi i)^{k}}{(k-1) ! \zeta(k)} \sum_{n=1}^{\infty} \sigma_{k-1}(n) q^{n}
$$

be the normalized Eisenstein series of weight $k$ for $\Gamma=\mathrm{SL}_{2}(\mathbb{Z})$, where $q=e^{2 \pi i z}$. The identities

$$
E_{4} \cdot E_{4}=E_{8}, \quad E_{4} \cdot E_{6}=E_{10}, \quad E_{4} \cdot E_{10}=E_{14}, \quad E_{6} \cdot E_{8}=E_{14}
$$

are well-known and follow from the fact that the vector space of modular forms of level 1 and weight $k$ is 1 -dimensional for $k \in\{4,6,8,10,14\}$. Elementary proofs of these identities can be found in [7].

Comparing Fourier coefficients of the power series in the products, we get interesting identities involving the divisor functions $\sigma_{k}(n)=\sum_{d \mid n} d^{k}$. For example, $E_{4} \cdot E_{4}=E_{8}$ gives

$$
\sigma_{7}(n)=\sigma_{3}(n)+120 \sum_{m=1}^{n-1} \sigma_{3}(m) \sigma_{3}(n-m) .
$$

Similar identities can be found by comparing coefficients in the other products.

Let $\Delta_{l}$ denote the unique cuspidal eigenform of weight $l$ in $\mathcal{S}_{l}(\Gamma)$ for $l \in\{12,16,18,20,22,26\}$. Along with the identities in (1) we have

$$
\begin{aligned}
& E_{4} \cdot \Delta_{12}=\Delta_{16}, \quad E_{4} \cdot \Delta_{16}=\Delta_{20}, \quad E_{4} \cdot \Delta_{18}=\Delta_{22}, \\
& E_{4} \cdot \Delta_{22}=\Delta_{26}, \quad E_{6} \cdot \Delta_{12}=\Delta_{18}, \quad E_{6} \cdot \Delta_{16}=\Delta_{22}, \\
& E_{6} \cdot \Delta_{20}=\Delta_{26}, \quad E_{8} \cdot \Delta_{12}=\Delta_{20}, \quad E_{8} \cdot \Delta_{18}=\Delta_{26}, \\
& E_{10} \cdot \Delta_{12}=\Delta_{22}, \quad E_{10} \cdot \Delta_{16}=\Delta_{26}, \quad E_{14} \cdot \Delta_{12}=\Delta_{26} \text {. }
\end{aligned}
$$


These give all cases where the product of two Hecke eigenforms is an eigenform. This was proven both by Duke in [2] and by Ghate in [4] using the Rankin-Selberg method. That is, the product of two Hecke eigenforms is a Hecke eigenform if and only if it is forced to be by dimension considerations. In [3] the first author found all cases where the product of two Hecke eigenforms is another Hecke eigenform for the congruence subgroup $\Gamma_{0}(p)$, for $p \geq 5$ prime.

It is still possible that the product of more than two eigenforms is an eigenform. In particular, if the product of two or more Eisenstein series is another Eisenstein series, then we may obtain identities similar to (2). The goal of this paper is to show that this does not happen. That is, the product of an arbitrary number of Hecke eigenforms is never a Hecke eigenform except in the cases obtained from (1) and (3). Note that $E_{4}^{2} \cdot E_{6}=E_{14}$, but this is trivially obtained from the identities $E_{4} \cdot E_{4}=E_{8}$ and $E_{6} \cdot E_{8}=E_{14}$. Other such products are obtained similarly.

In the first cases we prove that the product of an arbitrary number of Eisenstein series is never an Eisenstein series except when trivially obtained from (1). We consider products of the form

$$
E_{k_{1}} \cdots E_{k_{n}}=E_{l}
$$

where $l=k_{1}+\cdots+k_{n}$ and $n>1$. Assume that $k_{i} \leq k_{j}$ when $i<j$.

THEOREM 1. Let $E_{k}$ be the normalized Eisenstein series of weight $k$ for the full modular group $\Gamma$. If

$$
E_{k_{1}} \cdots E_{k_{n}}=E_{l}
$$

then $l \in\{8,10,14\}$.

The proof uses the nature of the zeros of the Eisenstein series and the growth of the coefficients

$$
C_{k}=\frac{(2 \pi i)^{k}}{\zeta(k)(k-1) !}
$$

The second case is to prove that the product of a cuspidal eigenform $f \in \mathcal{S}_{l}(\Gamma)$ and an arbitrary number of Eisenstein series is not a cuspidal eigenform.

THEOREM 2. Let $E_{k}$ be the normalized Eisenstein series of weight $k$ for the full modular group $\Gamma$ and let $f$ be a Hecke eigenform in $\mathcal{S}_{l}(\Gamma)$. If

$$
f \cdot E_{k_{1}} \cdots E_{k_{n}}
$$

is a Hecke eigenform then $l+\sum_{i=1}^{n} k_{i} \in\{16,18,20,22,26\}$.

Note that this is sufficient to prove our claim since if more than one of the factors is a cuspform, then the product cannot be an eigenform. 
2. Proof of Theorem 1. Note that repeat factors can occur as in $E_{4}^{2}=E_{8}$ and $E_{4}^{2} \cdot E_{6}=E_{14}$. The first lemma states that only $E_{4}$ can occur as a repeat factor and it can only occur with multiplicity two.

LEMMA 1. If $E_{k_{1}} \cdots E_{k_{n}}=E_{l}$ where $k_{1} \leq \cdots \leq k_{n}$, then

$$
k_{1}<\cdots<k_{n} \text { or } 4=k_{1}=k_{2}<k_{3}<\cdots<k_{n} .
$$

Proof. Let $v_{z}(f)$ be the order of vanishing of the modular form $f$ at the point $z$. We have

$$
v_{\infty}(f)+\frac{1}{2} v_{i}(f)+\frac{1}{3} v_{\varrho}(f)+\sum_{z}^{\prime} v_{z}(f)=\frac{k}{12},
$$

where the sum is over the points in the fundamental domain other than $i$ and $\varrho=e^{2 \pi i / 3}$ and $k$ is the weight of the modular form [5]. If we write $k=12 r+s$, where $r$ is an integer and $s \in\{0,4,6,8,10,14\}$, then $E_{k}(z)$ has exactly $r$ zeros on the unit circle between $i$ and $\varrho$, and each of these zeros is a simple zero [6].

Let $a_{r}$ be the number of $k_{i}$ 's congruent to $r$ modulo 12 . Noting that $E_{k}(i)=0$ if and only if $k \equiv 2(\bmod 4)$ and that $i$ is a simple zero, we have

$$
a_{2}+a_{6}+a_{10}= \begin{cases}0 & \text { if } l \equiv 0(\bmod 4), \\ 1 & \text { if } l \equiv 2(\bmod 4) .\end{cases}
$$

In particular, we cannot have repeated factors of $E_{k}$ for $k \equiv 2,6,10(\bmod 12)$.

Similarly, if we note that $E_{k}(\varrho)=0$ if and only if $k \equiv 2,4(\bmod 6)$, and the zero is a simple zero when $k \equiv 4(\bmod 6)$ and a double zero when $k \equiv 2$ $(\bmod 6)$, then we have

$$
2 a_{2}+a_{4}+2 a_{8}+a_{10}= \begin{cases}0 & \text { if } l \equiv 0(\bmod 6), \\ 1 & \text { if } l \equiv 4(\bmod 6) \\ 2 & \text { if } l \equiv 2(\bmod 6)\end{cases}
$$

In particular, we cannot have repeated factors of $E_{k}$ for $k \equiv 2,8(\bmod 12)$, and we cannot have more than two factors of $E_{k}$ for $k \equiv 4(\bmod 12)$.

It remains to show that there cannot be any repeat factors of $E_{k}$ for $k \equiv 0,4(\bmod 12)$ and $k \geq 12$. This follows from the fact that $E_{k}\left(e^{i \theta}\right)$ has the required number of zeros on the interval $\theta \in(\pi / 2,2 \pi / 3)$, and each of these zeros is a simple zero.

Recall that the Fourier expansion of the Eisenstein series is

$$
E_{k}(z)=1+C_{k} \sum_{n=1}^{\infty} \sigma_{k-1}(n) q^{n}
$$

where $C_{k}=(2 \pi i)^{k} / \zeta(k)(k-1)$ !. If we have

$$
E_{k_{1}} \cdots E_{k_{n}}=E_{l}
$$


then by comparing the $q$-coefficient in the product we have

$$
C_{k_{1}}+\cdots+C_{k_{n}}=C_{l} \text {. }
$$

The following two lemmas concern the growth of the coefficients $C_{k}$.

Lemma 2. If $10 \leq l<k$ or $l=8 \& k \geq 12$ or $l=6 \& k \geq 14$ or $l=4 \& k \geq 20$ then

$$
\left|C_{k}\right| \leq 2^{(l-k) / 2}\left|C_{l}\right|
$$

Proof. As

$$
1 \leq \zeta(k) \leq 1+\int_{1}^{\infty} x^{-k} d x=\frac{k}{k-1}
$$

for $k$ even, we have

$$
\frac{\left|C_{k+2}\right|}{\left|C_{k}\right|} \leq \frac{(2 \pi)^{2} \zeta(k)}{k(k+1) \zeta(k+2)} \leq \frac{(2 \pi)^{2}}{(k+1)(k-1)}
$$

Therefore for $k \geq 10,\left|C_{k+2}\right|<\frac{1}{2}\left|C_{k}\right|$. By induction, the result holds for all $k$.

We obtain the other cases by examining the list

$$
\begin{aligned}
& C_{4}=240, \quad C_{6}=-504, \quad C_{8}=480, \quad C_{10}=-264, \quad C_{12}=\frac{65520}{691}, \\
& C_{14}=-24, \quad C_{16}=\frac{16320}{3617}, \quad C_{18}=-\frac{28728}{43867}, \quad C_{20}=\frac{13200}{174611} .
\end{aligned}
$$

Lemma 3. The series $\sum_{j=2}^{\infty} C_{2 j}$ is absolutely convergent and for $m \geq 5$ we have

$$
\sum_{j=m}^{\infty}\left|C_{2 j}\right|<2\left|C_{2 m}\right|
$$

Proof. Absolute convergence follows immediately from Lemma 2 and the ratio test. For the upper bound,

$$
\begin{aligned}
\sum_{j=m}^{\infty}\left|C_{2 j}\right| & =\left|C_{2 m}\right|+\left|C_{2 m+2}\right|+\left|C_{2 m+4}\right|+\cdots \\
& <\left|C_{2 m}\right|+\frac{1}{2}\left|C_{2 m}\right|+\frac{1}{4}\left|C_{2 m}\right|+\cdots \\
& =\left|C_{2 m}\right|\left(1+\frac{1}{2}+\frac{1}{4}+\cdots\right)=2\left|C_{2 m}\right|
\end{aligned}
$$

The following lemma establishes that in all but a finite number of cases the product of an arbitrary number of Eisenstein series is not an Eisenstein series.

LEMma 4. If $k_{1} \geq 10$ or $k_{1} \in\{4,6,8\}$ and $k_{2} \geq 12$ then

$$
E_{k_{1}} \cdots E_{k_{n}} \neq E_{l} \text {. }
$$


Proof. Suppose that

$$
E_{k_{1}} \cdots E_{k_{n}}=E_{l} \text {. }
$$

Equating the $q$-coefficients we have

$$
C_{k_{1}}+\cdots+C_{k_{n}}=C_{l} \text {. }
$$

For $k_{1} \geq 10$, we find from Lemma 1 that the $k_{i}$ are distinct and therefore

$$
\begin{aligned}
\left|C_{k_{1}}\right| & \leq\left|C_{k_{2}}\right|+\left|C_{k_{3}}\right|+\cdots+\left|C_{l}\right| \\
& <\left|C_{k_{1}}\right|\left(2^{\left(k_{1}-k_{2}\right) / 2}+2^{\left(k_{1}-k_{3}\right) / 2}+\cdots+2^{\left(k_{1}-l\right) / 2}\right) \\
& <\left|C_{k_{1}}\right|\left(\frac{1}{2}+\frac{1}{4}+\frac{1}{8}+\cdots\right)=\left|C_{k_{1}}\right|,
\end{aligned}
$$

which is a contradiction.

For $k_{1} \in\{4,6,8\}$ and $k_{2} \geq 12$ we have

$$
\left|C_{k_{1}}\right| \leq\left|C_{k_{2}}\right|+\left|C_{k_{3}}\right|+\cdots+\left|C_{l}\right|<\sum_{m=k_{2} / 2}^{\infty}\left|C_{2 m}\right|<2\left|C_{k_{2}}\right|<200 .
$$

However, for $k_{1} \in\{4,6,8\}$ we have $\left|C_{k_{1}}\right| \geq 240$.

The remaining cases are

$$
\left(k_{1}, k_{2}\right) \in\{(4,4),(4,6),(4,8),(4,10),(6,8),(6,10),(8,10)\} .
$$

The cases $\left(k_{1}, k_{2}\right)=(4,8)$ or $(8,10)$ cannot happen, since this would contradict (5). That is, the order of vanishing at $\varrho=e^{2 \pi i / 3}$ would be too great to be an Eisenstein series. Similarly, the case $\left(k_{1}, k_{2}\right)=(6,10)$ cannot happen as this would contradict (4). That is, the order of vanishing at $i$ would be too great.

Note that $\left(k_{1}, k_{2}\right) \in\{(4,4),(4,6),(4,10),(6,8)\}$ occur in (1). It remains to show that there are no other such identities with these as factors.

Lemma 5. Let

$$
E_{k_{1}} \cdots E_{k_{n}}=E_{l}
$$

where $\left(k_{1}, k_{2}\right) \in\{(4,4),(4,6),(4,10),(6,8)\}$. If $\left(k_{1}, k_{2}\right) \in\{(4,6),(4,10)$, $(6,8)\}$ then $n=2$ and we have $E_{4} \cdot E_{6}=E_{10}, E_{4} \cdot E_{10}=E_{14}$, or $E_{6} \cdot E_{8}=E_{14}$. If $k_{1}=k_{2}=4$ then either $n=2$ and we have $E_{4} \cdot E_{4}=E_{8}$, or $n=3$ and we have $E_{4} \cdot E_{4} \cdot E_{6}=E_{14}$.

Proof. First consider $\left(k_{1}, k_{2}\right)=(6,8)$. Suppose that

$$
E_{6} \cdot E_{8} \cdot E_{k_{3}} \cdots E_{k_{n}}=E_{l} \text {. }
$$

Rewrite the above equation by substituting $E_{14}$ for $E_{6} \cdot E_{8}$. Note that none of the other factors can be $E_{14}$ by Lemma 1 . So this equation can be rewritten with the smallest factor greater than or equal to $E_{10}$, but we have shown this cannot happen. Therefore, there can be no other factors in the product, 
and $\left(k_{1}, k_{2}\right)=(6,8)$ implies that the only identity is $E_{6} \cdot E_{8}=E_{14}$. The other cases are handled similarly.

This finishes the proof of Theorem 1.

3. Proof of Theorem 2. We begin with a preliminary lemma.

Lemma 6. Let $f$ be a cusp form and a Hecke eigenform of weight l for $\Gamma$. If $f \cdot E_{k_{1}} \cdots E_{k_{n}}$ is an eigenform then $k_{j} \in\{4,6,8,10,14\}$ for $1 \leq j \leq n$.

Proof. Let $f \cdot g=h$, where $f=\sum_{m=1}^{\infty} a_{m} q^{m}$ with $a_{1}=1, g=1+$ $\sum_{m=1}^{\infty} \gamma_{m} q^{m}$ and $h=\sum_{m=1}^{\infty} b_{m} q^{m}$ with $a_{j}$ and $b_{j}$ algebraic integers for all $j$. Since $\gamma_{1}=b_{2}-a_{2}$ and $\gamma_{m}=b_{m+1}-a_{m+1}-a_{m} \gamma_{1}-a_{m-1} \gamma_{2}-\cdots-a_{2} \gamma_{m-1}$ for $m \geq 2$, by induction it follows that $\gamma_{j}$ is also an algebraic integer for every $j$.

As $f$ and $f \cdot E_{k_{1}} \cdots E_{k_{n}}$ are eigenforms, they have algebraic integer coefficients. From the previous paragraph, we deduce that $E_{k_{1}} \cdots E_{k_{n}}$ also has algebraic integer coefficients. Since each $E_{k_{j}}$ has rational Fourier coefficients, it follows that $E_{k_{1}} \cdots E_{k_{n}}$ has Fourier coefficients in $\mathbb{Z}$.

Let $p$ be the largest prime that occurs in the denominators of the reduced forms of all of the $C_{k_{j}}$ 's and suppose exactly $m$ of these $C_{k_{j}}$ 's have a factor of $p$ in the denominator. Without loss of generality we can suppose that these are $C_{k_{1}}, \ldots, C_{k_{m}}$. The $q^{m}$ th Fourier coefficient of $E_{k_{1}} \cdots E_{k_{n}}$ is

$$
\begin{gathered}
\sum_{j} C_{k_{j}} \sigma_{k_{j}-1}(m)+\cdots+\sum_{\substack{j_{1}, \ldots, j_{r} \\
m_{1}+\cdots+m_{r}=m}} C_{k_{j_{1}}} \cdots C_{k_{j_{r}}} \sigma_{k_{j_{1}-1}}\left(m_{1}\right) \cdots \sigma_{k_{j_{r}-1}}\left(m_{r}\right) \\
+\cdots+\sum_{j_{1}, \ldots, j_{m}} C_{k_{j_{1}}} \cdots C_{k_{j_{m}}} .
\end{gathered}
$$

If $p^{r}$ is the highest power of $p$ that occurs in the denominators of all of the above terms then only $C_{k_{1}} \cdots C_{k_{m}}$ has a factor of $p^{r}$ in the denominator. It follows that the reduced form of the rational number (6) must have a factor of $p^{r}$ in its denominator, and so cannot be an integer. Therefore we must have $C_{k_{j}} \in \mathbb{Z}$ for every $j$ and the lemma follows from Lemma 2 .

Hence we may consider products of the form $h=f \cdot E_{4}^{a} \cdot E_{6}^{b} \cdot E_{8}^{c} \cdot E_{10}^{d} \cdot E_{14}^{e}$. Since we have the identities in (1), we only need to consider products of the form $h=f \cdot E_{4}^{b} \cdot E_{6}^{c}$, where $f$ is a cusp form in $\mathcal{S}_{l}(\Gamma)$.

Note that $E_{4}^{b}=1+\sum_{n=1}^{\infty} b_{n} q^{n}$, where

$$
\begin{aligned}
& b_{1}=240 b, \\
& b_{2}=240\left(120 b^{2}-111 b\right), \\
& b_{3}=240\left(9600 b^{3}-26640 b^{2}+17068 b\right),
\end{aligned}
$$


and $E_{6}^{c}=1+\sum_{n=1}^{\infty} c_{n} q^{n}$, where

$$
\begin{aligned}
& c_{1}=-504 c, \\
& c_{2}=504\left(252 c^{2}-285 c\right), \\
& c_{3}=-504\left(42336 c^{3}-143640 c^{2}+101548 c\right) .
\end{aligned}
$$

We can expand $h=f \cdot E_{4}^{b} \cdot E_{6}^{c}=\left(\sum_{n=1}^{\infty} a_{n} q^{n}\right)\left(1+\sum_{n=1}^{\infty} b_{n} q^{n}\right)\left(1+\sum_{n=1}^{\infty} c_{n} q^{n}\right)$ as

$$
\begin{aligned}
h= & q+\left(a_{2}+\left(b_{1}+c_{1}\right)\right) q^{2}+\left(a_{3}+a_{2}\left(b_{1}+c_{1}\right)+\left(b_{2}+b_{1} c_{1}+c_{2}\right)\right) q^{3} \\
& +\left(a_{4}+a_{3}\left(b_{1}+c_{1}\right)+a_{2}\left(b_{2}+b_{1} c_{1}+c_{2}\right)+\left(b_{3}+b_{2} c_{1}+b_{1} c_{2}+c_{3}\right)\right) q^{4} \\
& +\cdots
\end{aligned}
$$

Since $h$ is an eigenform for the weight $l+4 b+6 c$ operator $T(2)$ and $f$ is an eigenform for the weight $l$ operator $T(2)$, we have

$$
\begin{aligned}
& \left(a_{2}+\left(b_{1}+c_{1}\right)\right)^{2}-2^{l+4 b+6 c-1} \\
& \quad=a_{2}^{2}-2^{l-1}+a_{3}\left(b_{1}+c_{1}\right)+a_{2}\left(b_{2}+b_{1} c_{1}+c_{2}\right)+\left(b_{3}+b_{2} c_{1}+b_{1} c_{2}+c_{3}\right) .
\end{aligned}
$$

Solving for $2^{l}$ we obtain

$$
\begin{aligned}
2^{l}= & \frac{2}{2^{4 b+6 c}-1}\left(a_{3}\left(-b_{1}-c_{1}\right)+a_{2}\left(2 b_{1}+2 c_{1}-b_{2}-b_{1} c_{1}-c_{2}\right)\right. \\
& \left.+\left(b_{1}+c_{1}\right)^{2}-\left(b_{3}+b_{2} c_{1}+b_{1} c_{2}+c_{3}\right)\right) .
\end{aligned}
$$

From the Ramanujan-Petersson estimate $\left|a_{p}\right| \leq 2 p^{(l-1) / 2}$ (see [1]) we have

$$
\begin{aligned}
2^{l} \leq & \frac{2}{2^{4 b+6 c}-1}\left(2 \cdot 3^{(l-1) / 2}\left|b_{1}+c_{1}\right|\right. \\
& +2 \cdot 2^{(l-1) / 2}\left|2 b_{1}+2 c_{1}-b_{2}-b_{1} c_{1}-c_{2}\right| \\
& \left.+\left|\left(b_{1}+c_{1}\right)^{2}-\left(b_{3}+b_{2} c_{1}+b_{1} c_{2}+c_{3}\right)\right|\right) .
\end{aligned}
$$

Therefore $l$ must satisfy at least one of the following inequalities:

$$
\begin{aligned}
\left(\frac{2}{\sqrt{3}}\right)^{l} \leq & \frac{12}{\sqrt{3}\left(2^{4 b+6 c}-1\right)}\left|b_{1}+c_{1}\right|=\frac{12}{\sqrt{3}\left(2^{4 b+6 c}-1\right)}|240 b-504 c| \\
(\sqrt{2})^{l} \leq & \frac{2^{3 / 2} \cdot 3}{2^{4 b+6 c}-1}\left|2 b_{1}+2 c_{1}-b_{2}-b_{1} c_{1}-c_{2}\right| \\
= & \frac{2^{3 / 2} \cdot 3}{2^{4 b+6 c}-1} \mid 27120 b+142632 c-28800 b^{2} \\
& +120960 b c-127008 c^{2} \mid
\end{aligned}
$$




$$
\begin{aligned}
2^{l} \leq & \frac{2 \cdot 3}{2^{4 b+6 c}-1}\left|\left(b_{1}+c_{1}\right)^{2}-\left(b_{3}+b_{2} c_{1}+b_{1} c_{2}+c_{3}\right)\right| \\
= & \frac{2 \cdot 3}{2^{4 b+6 c}-1} \mid 6451200 b^{2}+20805120 b c-72140544 c^{2} \\
& -4096320 b-2304000 b^{3}+14515200 c b^{2}-30481920 b c^{2} \\
& +51180192 c+21337344 c^{3} \mid .
\end{aligned}
$$

If we let $x=\max \{b, c\}$, then we have

$$
l \leq \sup \left\{\frac{\log \left(\frac{6048 x}{\sqrt{3}\left(16^{x}-1\right)}\right)}{\log (2 / \sqrt{3})}, \frac{\log \left(\frac{2^{3 / 2} \cdot 1085598 x^{2}}{16^{x}-1}\right)}{\log (\sqrt{2})}, \frac{\log \left(\frac{792836144 x^{3}}{16^{x}-1}\right)}{\log (2)}\right\} .
$$

If $x \geq 7$ then $l<12$, and since there are no cusp forms of weight $l$ for $l<12$, there are no solutions. So we only have the cases where $E_{4}^{b} \cdot E_{6}^{c}$ with $b, c \leq 6$. By substituting directly into inequalities (7), (8), and (9) we see that $l<12$ in all cases except

$$
(b, c) \in\{(0,1),(0,2),(1,0),(1,1),(1,2),(2,0),(2,1),(3,0),(4,0)\} .
$$

The cases $(b, c) \in\{(0,1),(1,0),(1,1),(2,0),(2,1)\}$ were handled in [2]. And so we are left with the cases $(b, c)=(0,2)$ where $l<18,(b, c)=(1,2)$ where $l<13,(b, c)=(0,3)$ where $l<18$, and $(b, c)=(0,4)$ where $l<13$. These cases give the possible eigenforms

$\Delta_{12} \cdot E_{6}^{2}, \quad \Delta_{16} \cdot E_{6}^{2}, \quad \Delta_{12} \cdot E_{6}^{2} \cdot E_{4}, \quad \Delta_{12} \cdot E_{4}^{3}, \quad \Delta_{16} \cdot E_{4}^{3}, \quad \Delta_{12} \cdot E_{4}^{4}$.

The products $\Delta_{16} \cdot E_{6}^{2}$ and $\Delta_{12} \cdot E_{6}^{2} \cdot E_{4}$ are the same, as are the products $\Delta_{16} \cdot E_{4}^{3}$ and $\Delta_{12} \cdot E_{4}^{4}$. So we are left with checking if the products

$$
\begin{aligned}
\Delta_{12} \cdot E_{6}^{2} & =q-1032 q^{2}+245196 q^{3}+10965568 q^{4}+\cdots, \\
\Delta_{12} \cdot E_{6}^{2} \cdot E_{4} & =q-792 q^{2}-324 q^{4}+67590208 q^{4}+\cdots, \\
\Delta_{12} \cdot E_{4}^{3} & =q+696 q^{2}+162252 q^{3}+12831808 q^{4}+\cdots, \\
\Delta_{12} \cdot E_{4}^{4} & =q+936 q^{2}+331452 q^{3}+53282368 q^{4}+\cdots
\end{aligned}
$$

are eigenforms. Since these products are not eigenvectors of the Hecke operator $T(2)$, they are not Hecke eigenforms.

Acknowledgements. The authors would like to thank the anonymous referee for the corrections and stylistic suggestions.

\section{References}

[1] P. Deligne, La conjecture de Weil. I, Inst. Hautes Études Sci. Publ. Math. 43 (1974), 273-307.

[2] W. Duke, When is the product of two Hecke eigenforms an eigenform?, in: Number Theory in Progress, Vol. 2 (Zakopane-Kościelisko, 1997), de Gruyter, Berlin, 1999, $737-741$. 
[3] B. A. Emmons, Products of Hecke eigenforms, J. Number Theory 115 (2005), 381393.

[4] E. Ghate, On products of eigenforms, Acta Arith. 102 (2002), 27-44.

[5] A. W. Knapp, Elliptic Curves, Princeton Univ. Press, Princeton, NJ, 1992.

[6] F. K. C. Rankin and H. P. F. Swinnerton-Dyer, On the zeros of Eisenstein series, Bull. London Math. Soc. 2 (1970), 169-170.

[7] R. A. Rankin, Elementary proofs of relations between Eisenstein series, Proc. Roy. Soc. Edinburgh Sect. A 76 (1976/77), 107-117.

Department of Mathematics

Utica College

1600 Burrstone Road

Utica, NY 13502, U.S.A.

E-mail: bemmons@utica.edu
Department of Mathematics Western Kentucky University 1906 College Heights Blvd. \#11078 Bowling Green, KY 42101-1078, U.S.A. E-mail: dominic.lanphier@wku.edu

Received on 29.12.2006

and in revised form on 6.9.2007 